\title{
Proton decoupling and recoupling under double-nutation irradiation in solid-state NMR.
}

\author{
AUTHOR(S): \\ Takeda, Kazuyuki; Wakisaka, Asato; Takegoshi, K
}

\section{CITATION:}

Takeda, Kazuyuki ... [et al]. Proton decoupling and recoupling under double-nutation irradiation in solid-state NMR.. The Journal of chemical physics 2014, 141(22): 224202.

\section{ISSUE DATE:}

2014-12-14

URL:

http://hdl.handle.net/2433/193245

\section{RIGHT:}

Copyright 2014 American Institute of Physics. This article may be downloaded for personal use only. Any other use requires prior permission of the author and the American Institute of Physics 


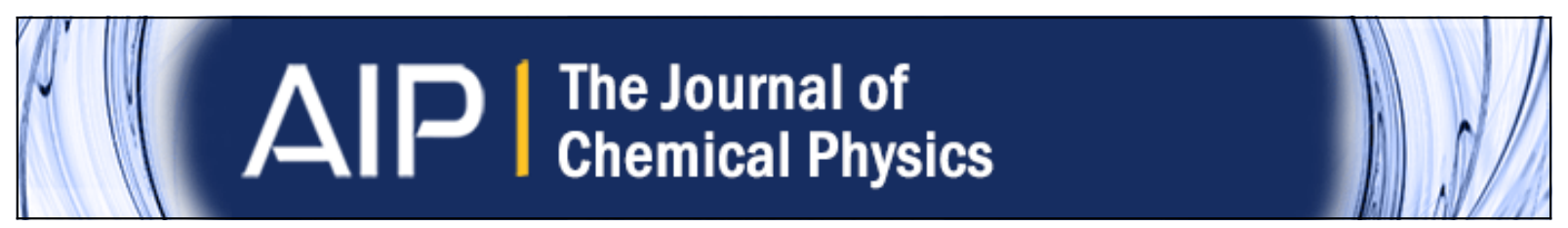

Proton decoupling and recoupling under double-nutation irradiation in solid-state NMR

Kazuyuki Takeda, Asato Wakisaka, and K. Takegoshi

Citation: The Journal of Chemical Physics 141, 224202 (2014); doi: 10.1063/1.4903173

View online: http://dx.doi.org/10.1063/1.4903173

View Table of Contents: http://scitation.aip.org/content/aip/journal/jcp/141/22?ver=pdfcov

Published by the AIP Publishing

\section{Articles you may be interested in}

Mechanism of dilute-spin-exchange in solid-state NMR

J. Chem. Phys. 140, 124201 (2014); 10.1063/1.4869345

Refocused continuous-wave decoupling: A new approach to heteronuclear dipolar decoupling in solid-state NMR spectroscopy

J. Chem. Phys. 137, 214202 (2012); 10.1063/1.4768953

Unexpected effects of third-order cross-terms in heteronuclear spin systems under simultaneous radio-frequency irradiation and magic-angle spinning NMR

J. Chem. Phys. 136, 084503 (2012); 10.1063/1.3684879

Supercycled homonuclear dipolar decoupling in solid-state NMR: Toward cleaner H 1 spectrum and higher spinning rates

J. Chem. Phys. 128, 052309 (2008); 10.1063/1.2834730

Decoupling and recoupling using continuous-wave irradiation in magic-angle-spinning solid-state NMR: A unified description using bimodal Floquet theory

J. Chem. Phys. 123, 064102 (2005); 10.1063/1.1944291

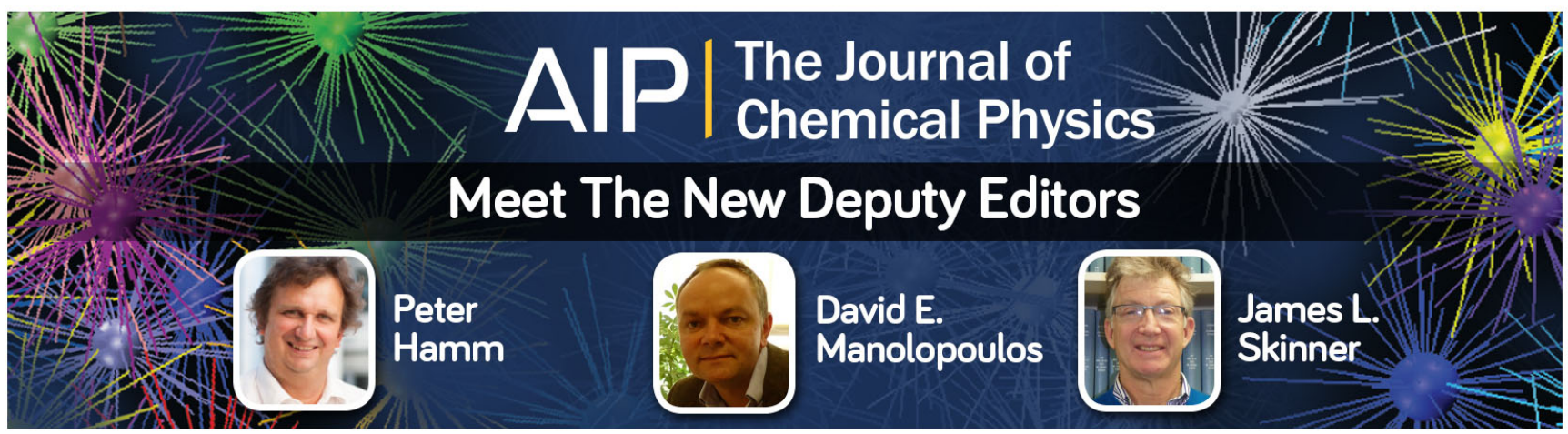




\title{
Proton decoupling and recoupling under double-nutation irradiation in solid-state NMR
}

\author{
Kazuyuki Takeda, ${ }^{\text {a) }}$ Asato Wakisaka, and K. Takegoshi \\ Division of Chemistry, Graduate School of Science, Kyoto University, 606-8502 Kyoto, Japan
}

(Received 17 July 2014; accepted 17 November 2014; published online 10 December 2014)

\begin{abstract}
The effect of ${ }^{1} \mathrm{H}$ decoupling in magic-angle spinning solid-state NMR is studied under radiofrequency irradiation causing simultaneous nutations around a pair of orthogonal axes. Double-nutation with an arbitrary pair of nutation frequencies is implemented through modulation of the amplitude, phase, and frequency of the transmitting pulses. Similarity and difference of double-nutation decoupling and two-pulse phase-modulation decoupling schemes [A. E. Bennett, C. M. Rienstra, M. Auger, K. V. Lakshmi, and R. G. Griffin, J. Chem. Phys. 103, 6951-6958 (1995) and I. Scholz, P. Hodgkinson, B. H. Meier, and M. Ernst, J. Chem. Phys. 130, 114510 (2009)] are discussed. The structure of recoupling bands caused by interference of the ${ }^{1} \mathrm{H}$ spin nutation with sample spinning is studied by both experiments and numerical simulations. () 2014 AIP Publishing LLC. [http://dx.doi.org/10.1063/1.4903173]
\end{abstract}

\section{INTRODUCTION}

In nuclear magnetic resonance (NMR) of organic solids, ${ }^{1} \mathrm{H}$ decoupling ${ }^{1,2}$ is essential to obtain highly resolved spectra of rare spins, such as ${ }^{13} \mathrm{C}$ and ${ }^{15} \mathrm{~N}$. Its key is to apply radiofrequency (rf) irradiation to the ${ }^{1} \mathrm{H}$ spins, so as to average out the dipolar interactions between the ${ }^{1} \mathrm{H}$ spins and the spins being observed. A straightforward and traditional way of applying continuous-wave (cw) rf irradiation ${ }^{3}$ has extensively been used until mid 1990s. However, its performance was found to become poor, and thus cw decoupling is used today only when the decoupling requirement is not demanding, or when the ${ }^{1} \mathrm{H}$ magnetization needs to be flipped back along the static magnetic field. ${ }^{4,5}$

In efforts to realize better decoupling performance, various rf-modulated schemes were developed. Tekely et al. proposed to apply periodic phase reversal of the decoupling rf field. ${ }^{6}$ This method, also known as $\mathrm{X}$ inverse-X (XiX), works well especially under fast magic angle spinning (MAS). ${ }^{7,8}$ Bennett et al. developed two-pulse phase modulation (TPPM) decoupling, ${ }^{9}$ in which a pair of phase-shifted pulses are applied one after another. Very recently, Vinther $e t$ al. proposed refocused continuous-wave (rCW) schemes, reporting that insertion of rotor synchronized $\pi$ pulses during $\mathrm{cw}$ irradiation can suppress residual couplings up to the third order. ${ }^{10,11}$ At present, TPPM and its variants, such as swept-frequency TPPM $\left(\mathrm{SW}_{\mathrm{f}}\right.$-TPPM $),{ }^{12}$ SPARC, ${ }^{13}$ SPINAL, ${ }^{14}$ cosine phase modulation (CPM), ${ }^{15}$ and so on, have found extensive applications.

The effect of TPPM on spin dynamics is diverse, depending on the choice of the three parameters: the rf amplitude $v_{\mathrm{rf}}$, phase angle $\phi_{0}$, and pulse duration $\tau_{\mathrm{p}}$ (Fig. 1). In the case of $\phi_{0}=0$, TPPM decoupling reduces to $\mathrm{cw}$ decoupling, while for $\phi_{0}=\pi / 2$ the sequence becomes equivalent to XiX. For relatively large $\phi_{0}(<\pi / 2)$, the sequence is given the name of high-phase TPPM. ${ }^{16,17}$

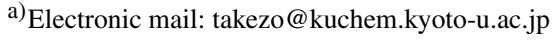

In general, under MAS and TPPM decoupling, the spin Hamiltonian acquires time dependence according to three incommensurable frequencies due to irradiation, phase modulation, and sample spinning. Scholz et al. exploited a triplemode Floquet theory to derive analytical expressions for the effective Hamiltonians carrying the residual couplings, which, in many cases, arise from the cross terms between anisotropic chemical shielding interactions of the irradiated spins and the relevant heteronuclear dipolar couplings. ${ }^{18}$ The cross terms were studied as a function of the phase shift $\phi_{0}$ and the pulse width $\tau_{\mathrm{p}}$ for various rf amplitudes $v_{\mathrm{rf}}$, and were shown to have minima along the line satisfying

$$
\tau_{\mathrm{p}}=\frac{\pi}{v_{\mathrm{rf}} \cos \phi_{0}},
$$

for such small $\phi_{0}$ that $\tan \phi_{0} \sim \phi_{0}$.

The reason why the condition given by Eq. (1) leads to better decoupling was accounted for by Gan and Ernst, ${ }^{19}$ who showed that simultaneous nutation of the ${ }^{1} \mathrm{H}$ spins around a pair of orthogonal axes contribute to second averaging of the residual couplings. That is, the rf Hamiltonian has, in addition to the major part causing $\mathrm{cw}$ nutation, a number of perturbative Fourier components rotating at frequencies $n \pi / \tau_{\mathrm{p}}$, where $n= \pm 1, \pm 2, \ldots$ When Eq. (1) is met, the $n=1$ term alone has an appreciable effect of nutating the spins about an axis orthogonal to the major cw field. Gan and Ernst performed left-handed circular frequency modulation and phase modulation (FMPM ${ }^{\mathrm{L}}$ ) and right-handed circular frequency modulation and phase modulation $\left(\mathrm{FMPM}^{\mathrm{R}}\right){ }^{19}$ Their result that the former leads to improved decoupling while the latter was ineffective proved that inclusion of double nutation is the key toward better decoupling. Indeed, the CPM scheme, in which the residual harmonics terms are absent except for the $n= \pm 1$ term, showed equivalent decoupling performance to that in TPPM. ${ }^{15,18}$ Extension of the idea of double nutation to include one more nutation was reported by Takegoshi et al., who showed that decoupling by 


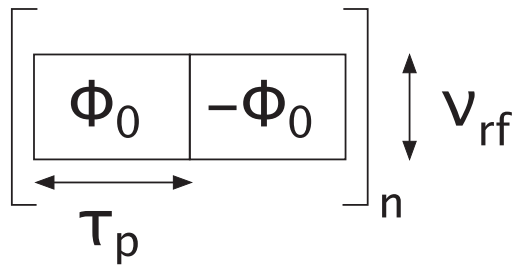

FIG. 1. A pulse sequence of TPPM. A pair of pulses with phases of $\phi_{0}$ and $-\phi_{0}$ are applied one after another. The pulse width and the amplitude are represented by $\tau_{\mathrm{p}}$ and $v_{\mathrm{rf}}$.

triple nutation is feasible using additional amplitude modulation, and that there is discernible line-narrowing effect due to the third-averaging effect. ${ }^{20}$ Such triple nutation, and even quadruple nutation, were also introduced in the context of spin-recoupling experiments. ${ }^{21,22}$

For double nutation that TPPM and its variants can perform, the second nutation frequency $v_{2}$ is inevitably much smaller than the first nutation frequency $v_{1}$, so that the case where $v_{1} \sim v_{2}$ or $v_{1}<v_{2}$ has not been explored. In this work, we complement the study of ${ }^{1} \mathrm{H}$ decoupling under double nutation to include general cases, i.e., with arbitrary combinations of the individual nutation frequencies $\left(v_{1}, v_{2}\right)$. Such general double-nutation decoupling can be implemented by simultaneous modulation of the rf amplitude, phase, and frequency. We show that, for relatively small second nutation frequencies, both double nutation and TPPM schemes perform equivalently, whereas discrepancy becomes prominent as increasing $v_{2}$.

Under MAS, careful choice of the TPPM parameters so as not to cause rotary resonance recoupling (R3) is of practical importance. Indeed, high-phase TPPM was developed to avoid degradation of the decoupling performance due to R3. ${ }^{16,17}$ So far, comparisons were made between high-phase TPPM and another R3-robust technique known as phaseinverted supercycled sequence for attenuation of rotary resonance (PISSARRO). ${ }^{23-26}$ In general, there exist a number of combinations of the experimental parameters that result in poor decoupling, and a set of such combination exhibits the recoupling bands to avoid. Scholz et al. studied the recoupling conditions extensively, covering the general TPPM cases. ${ }^{18}$ Here, we examine the recoupling bands of the double nutation scheme and make comparison with those in TPPM implemented in the double nutation regime, i.e., under the restriction given by Eq. (1). When the decoupling performance is plotted as a function of the TPPM parameters $v_{\mathrm{rf}}$ and $\phi_{0}$, the recoupling bands render somewhat complicated, curved lines. We show that, when the data are mapped into another plane spanned by the first and the second nutation frequencies $\left(v_{1}\right.$, $v_{2}$ ), all recoupling bands become simple straight lines. The result indicates that, even though the rf amplitude and the rf phase are the straightforward basis parameters in TPPM, the nutation frequencies $v_{1}$ and $v_{2}$ would give a natural basis set from the viewpoint of spin decoupling dynamics.

\section{THEORY}

Let us suppose that rf irradiation is applied at the abundant spins $I$ during observation of the transverse magnetiza- tion of the rare spin $S$. For a given set of amplitude $a(t)$, phase $\phi(t)$, and frequency $v(t)$ of the transmitted pulse, the rotatingframe representation of the rf Hamiltonian $\mathcal{H}_{\mathrm{rf}}$ is given by

$$
\mathcal{H}_{\mathrm{rf}}(t)=a(t)\left[I_{x} \cos \phi(t)+I_{y} \sin \phi(t)\right]+2 \pi \Delta v(t) I_{z} .
$$

Here, $\Delta v$ is the difference of the carrier frequency $v$ from the Larmor frequency $v_{0}$, i.e., $\Delta v=v-v_{0}$. In practice, it is often convenient to incorporate frequency modulation into phase modulation, so that

$$
\mathcal{H}_{\mathrm{rf}}(t)=a(t)\left[I_{x} \cos \phi^{\prime}(t)+I_{y} \sin \phi^{\prime}(t)\right],
$$

where

$$
\phi^{\prime}(t)=\phi(t)+2 \pi \int_{0}^{t} d t^{\prime} \Delta v\left(t^{\prime}\right)
$$

The rf propagator $U_{\mathrm{rf}}$ is related with $\mathcal{H}_{\mathrm{rf}}$ through

$$
U_{\mathrm{rf}}(t)=T \exp \left[-i \int d t \mathcal{H}_{\mathrm{rf}}(t)\right]
$$

or $^{27}$

$$
\mathcal{H}_{\mathrm{rf}}(t)=i\left(\frac{d U_{\mathrm{rf}}(t)}{d t}\right) U_{\mathrm{rf}}^{-1}(t)
$$

\section{A. Rf Hamiltonian for general double nutation}

We consider double nutation around the $-x$ and $-y$ axes with nutation frequencies of $v_{1}$ and $v_{2}$. The rf propagator $U_{\mathrm{rf}}^{\text {DONUT }}$ takes the following form:

$$
U_{\mathrm{rf}}^{\text {DONUT }}(t)=\exp \left[2 \pi i v_{2} t I_{y}\right] \exp \left[2 \pi i v_{1} t I_{x}\right] .
$$

Here, the superscript DONUT stands for DOuble NUTation. Using Eq. (6), the corresponding rf Hamiltonian $\mathcal{H}_{\mathrm{rf}}^{\text {DONUT }}$ is calculated to be

$$
\begin{aligned}
\mathcal{H}_{\mathrm{rf}}^{\text {DONUT }}(t)= & -2 \pi v_{1}\left[I_{x} \cos \left(2 \pi v_{2} t\right)+I_{z} \sin \left(2 \pi v_{2} t\right)\right] \\
& -2 \pi v_{2} I_{y} .
\end{aligned}
$$

By comparing Eq. (8) with Eq. (2), we obtain the amplitude $a(t)$, phase $\phi(t)$, and frequency offset $\Delta v(t)$ implementing pure double nutation as

$$
\begin{gathered}
a(t)=2 \pi\left[v_{1}^{2} \cos ^{2}\left(2 \pi v_{2} t\right)+v_{2}^{2}\right]^{\frac{1}{2}}, \\
\phi(t)=\tan ^{-1}\left[\frac{v_{2}}{v_{1} \cos \left(2 \pi v_{2} t\right)}\right],
\end{gathered}
$$

and

$$
\Delta v(t)=-v_{1} \sin \left(2 \pi v_{2} t\right) .
$$

Using Eq. (4), Eqs. (10) and (11) can be combined into

$$
\phi^{\prime}(t)=\phi(t)+\frac{v_{1}}{v_{2}}\left[\cos \left(2 \pi v_{2} t\right)-1\right]
$$

Figure 2 shows examples of the amplitude, phase, and frequency profiles required to implement double-nutation decoupling. 


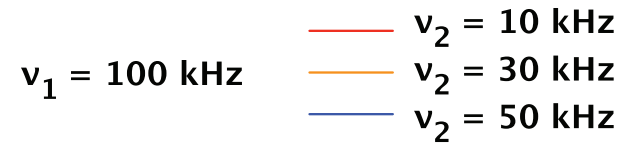

(a)

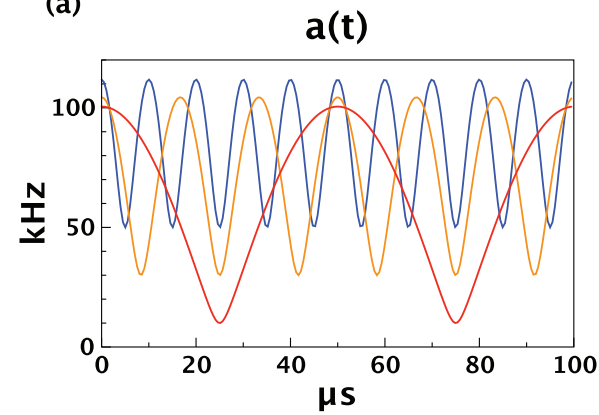

(c)

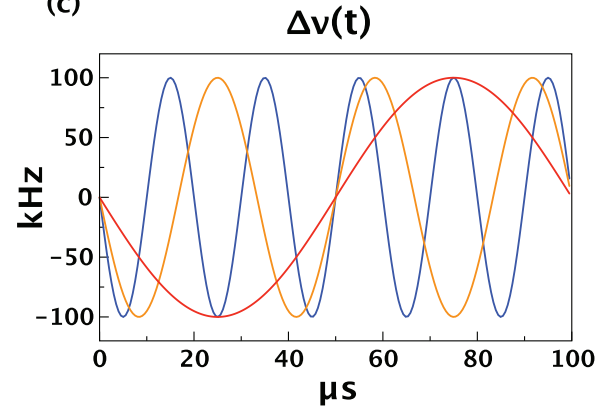

(b)

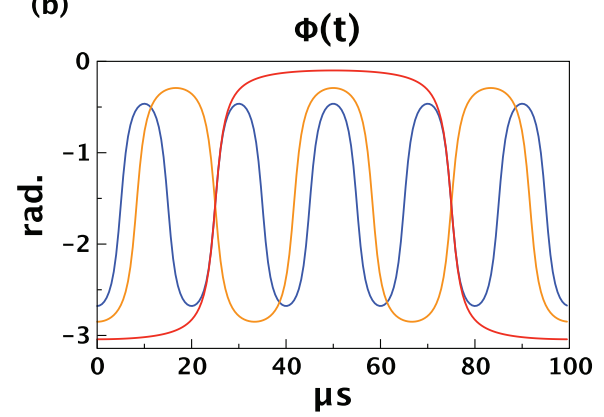

(d)

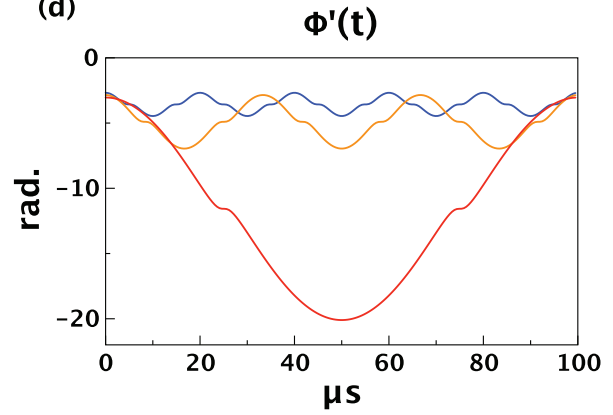

FIG. 2. Profiles of (a) amplitude, (b) phase, and (c) frequency modulation for implementing double-nutation irradiation with the first nutation frequency $v_{1}$ of $100 \mathrm{kHz}$ and the second nutation frequency $v_{2}$ of $10 \mathrm{kHz}$ (red lines), $30 \mathrm{kHz}$ (orange lines), and $50 \mathrm{kHz}$ (blue lines). The phase and frequency modulation can be implemented altogether using another phase modulation according to Eq. (12), as depicted in (d).

\section{B. Rf propagator under TPPM irradiation}

In the case of TPPM decoupling (Fig. 1), the amplitude $a(t)$ and the phase $\phi(t)$ of the rf Hamiltonian (Eq. (2)) are given by

$$
a(t)=2 \pi v_{\mathrm{rf}},
$$

and

$$
\phi(t)=\left\{\begin{array}{ll}
\phi_{0}, & 2 k \tau_{p} \leq t-t_{0}<(2 k+1) \tau_{p} \\
-\phi_{0}, & (2 k+1) \tau_{p} \leq t-t_{0}<(2 k+2) \tau_{p}
\end{array} .\right.
$$

We assume that the rf irradiation is applied on-resonance, so that $\Delta v(t)=0 . t_{0}$ is the origin of time, which may be chosen arbitrarily. Even though $t_{0}$ may be set zero and eliminated from Eq. (14), we leave it on purpose, because the expressions of the rf Hamiltonian and thereby that of the propagator depend on $t_{0}$ (vide infra). We, however, keep in mind that the choice of $t_{0}$ does not affect the spin decoupling dynamics.

Dividing $\mathcal{H}_{\mathrm{rf}}$ into time-independent and time-dependent terms, and using Fourier expansion, we write

$$
\begin{gathered}
\mathcal{H}_{\mathrm{rf}}=\mathcal{H}_{\mathrm{rf} 1}+\mathcal{H}_{\mathrm{rf} 2}, \\
\mathcal{H}_{\mathrm{rf} 1}=2 \pi v_{\mathrm{m}} I_{x},
\end{gathered}
$$

and

$$
\mathcal{H}_{\mathrm{rf} 2}=2 \pi v_{\mathrm{rf}} \sin \phi_{0} I_{y}
$$

$$
\cdot \frac{4}{\pi} \sum_{k=0}^{\infty} \frac{1}{2 k+1} \sin \left[2 \pi(2 k+1) v_{\mathrm{m}}\left(t-t_{0}\right)\right]
$$

where

$$
v_{\mathrm{m}}=v_{\mathrm{rf}} \cos \phi_{0} .
$$

The rf propagator is given by $U_{\mathrm{rf}}=U_{0} U_{1}$, where

$$
U_{0}=\exp \left[-i t \mathcal{H}_{\mathrm{rf} 1}\right]
$$

and

$$
U_{1}=T \exp \left[-i \int d t \tilde{\mathcal{H}}_{\mathrm{rf} 2}\right]
$$

with

$$
\begin{aligned}
\widetilde{\mathcal{H}}_{\mathrm{rf} 2}= & U_{0}^{-1} \mathcal{H}_{\mathrm{rf} 2} U_{0} \\
= & \left(I_{y} \cos 2 \pi v_{\mathrm{m}} t-I_{z} \sin 2 \pi v_{\mathrm{m}} t\right) 2 \pi v_{\mathrm{rf}} \sin \phi_{0} \\
& \times \frac{4}{\pi} \sum_{k=0}^{\infty} \frac{1}{2 k+1} \sin \left[2 \pi(2 k+1) v_{\mathrm{m}}\left(t-t_{0}\right)\right] .
\end{aligned}
$$

By taking a time average of Eq. (21) over a period $1 / v_{\mathrm{m}}$, we obtain

$$
\widetilde{\mathcal{H}}_{\mathrm{rf} 2}=-4 v_{\mathrm{rf}} \sin \phi_{0}\left[I_{z} \cos 2 \pi v_{\mathrm{m}} t_{0}+I_{y} \sin 2 \pi v_{\mathrm{m}} t_{0}\right] .
$$


We now approximate Eq. (20) by

$$
U_{1} \sim \exp \left[-i t \widetilde{\mathcal{H}}_{\mathrm{rf} 2}\right]
$$

By going into the interaction frame with respect to the rf Hamiltonian, the internal Hamiltonian is manipulated during a time interval from 0 to $t$ in such a way that the spin rotation is performed according to a unitary operator

$$
\begin{aligned}
U(0 ; t)= & U_{\mathrm{rf}}^{-1}=U_{1}^{-1} U_{0}^{-1} \\
= & \exp \left[2 \pi i v_{2} t\left(-I_{z} \cos 2 \pi v_{\mathrm{m}} t_{0}-I_{y} \sin 2 \pi v_{\mathrm{m}} t_{0}\right)\right] \\
& \times \exp \left[2 \pi i v_{1} t I_{x}\right],
\end{aligned}
$$

where

$$
v_{1}=v_{\mathrm{m}}=v_{\mathrm{rf}} \cos \phi_{0}
$$

and

$$
v_{2}=\frac{2}{\pi} v_{\mathrm{rf}} \sin \phi_{0}
$$

We now make an arbitrary choice of the time origin $t_{0}$ to be $-1 /\left(4 v_{\mathrm{m}}\right)$. Then, we have

$$
U(0 ; t)=\exp \left[2 \pi i v_{2} t I_{y}\right] \exp \left[2 \pi i v_{1} t I_{x}\right] .
$$

The effect of this propagator is to perform double nutation in the $I$ spin space. In the present case, the first and the second nutations are performed about the $-x$ and $-y$ axes. Note that the second nutation axis depends on the choice of the time origin $t_{0}$. For example, when $t_{0}=1 /\left(2 v_{\mathrm{m}}\right), U(0 ; t)$ $=e^{2 \pi i v_{2} t I_{z}} e^{2 \pi i v_{1} t I_{x}}$, so that the second nutation is done about the $-z$ axis. Importantly, for any $t_{0}$, the axes of the first and the second nutations are orthogonal.

Using a similar procedure, the TPFM and FMPM $^{\mathrm{L}}$ schemes that Gan and Ernst demonstrated ${ }^{19}$ can be described in terms of a double-nutation propagator of the form of Eq. (27). In FMPM ${ }^{\mathrm{R}}$, however, the sense of rotation of the perturbative part of the rf field in the rotating frame is opposite from that in $\mathrm{FMPM}^{\mathrm{L}}$, so that the interaction representation with respect to the stationary part of the rf Hamiltonian results in a propagator of a different form

$$
\exp \left[2 \pi i v_{2} t\left(I_{y} \cos 4 \pi v_{1} t+I_{z} \sin 4 \pi v_{1} t\right)\right] \exp \left[2 \pi i v_{1} t I_{x}\right] \text {. }
$$

Using Eqs. (25) and (26), correspondence between the parameter sets $\left(\phi_{0}, v_{\mathrm{rf}}\right)$ and $\left(v_{1}, v_{2}\right)$ can be made, and the coordinate system spanned by the TPPM parameter, namely, $\left(\phi_{0}, v_{\mathrm{rf}}\right)$, can be mapped into the plane spanned by the doublenutation frequencies $\left(v_{1}, v_{2}\right)$, as described in Fig. 3. By erasing $\phi_{0}$, we obtain

$$
v_{1}^{2}+\left(\frac{\pi}{2} v_{2}\right)^{2}=v_{\mathrm{rf}}^{2}
$$

Equation (29) indicates that, in the $v_{1}-v_{2}$ plane, the line of constant rf amplitude is described by an arc of an ellipse. (a)

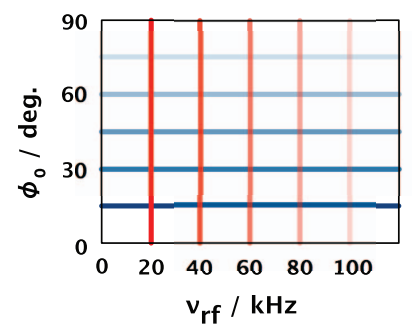

(b)

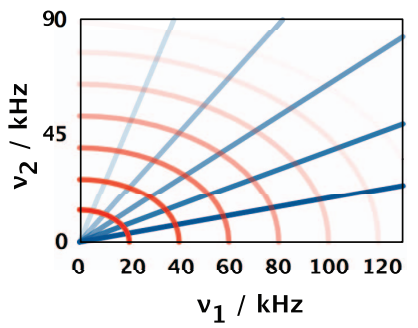

FIG. 3. Visualization of the relationship between the TPPM parameters $\left(v_{\mathrm{rf}}\right.$, $\left.\phi_{0}\right)$ and the frequencies $\left(v_{1}, v_{2}\right)$ of double nutation. (a) shows a plane spanned by the coordinate lines of constant $v_{\text {rf }}$ (red lines) and those of constant $\phi_{0}$ (blue lines). These coordinate lines are mapped into curved lines in another plane spanned by $\left(v_{1}, v_{2}\right)$, as shown in (b).

When $v_{\text {rf }}$ is erased using Eqs. (25) and (26), we obtain

$$
v_{2}=\frac{2}{\pi} \tan \phi_{0} v_{1}
$$

Thus, the constant-phase line is a linear function with a slope given by $(2 / \pi) \tan \phi_{0}$.

Even though the map operation itself can be performed over a wide range of the parameters, equivalence between TPPM and double nutation relies on the validity of the approximation we made in Eq. (22). When $v_{\mathrm{m}} \gg v_{\mathrm{rf}} \sin \phi_{0}$, or $\phi_{0}$ is small so that $\tan \phi_{0} \ll 1$, they are expected to give equivalent results, whereas for larger $\phi_{0}$ the effect of TPPM on spin nutation can no longer be viewed as double nutation.

\section{EXPERIMENTAL}

Experiments were performed in a polycrystalline sample of $2-{ }^{13} \mathrm{C}-{ }^{15} \mathrm{~N}$-labeled glycine in a magnetic field of $9.4 \mathrm{~T}$ at room temperature using a Chemagnetic $3.2 \mathrm{~mm}$ MAS probe and an Opencore NMR spectrometer. ${ }^{28,29}$ The carrier frequencies for the ${ }^{1} \mathrm{H}$ and ${ }^{13} \mathrm{C}$ channels were $400.2409 \mathrm{MHz}$ and $100.6505 \mathrm{MHz}$, respectively.

\section{RESULTS AND DISCUSSION}

Figures 4(a) and 4(b) show peak heights of the ${ }^{13} \mathrm{C}$ resonance line of the methylene carbon in polycrystalline 2${ }^{13} \mathrm{C},{ }^{15} \mathrm{~N}$-labeled glycine under ${ }^{1} \mathrm{H}$ double-nutation decoupling as a function of the first and second nutation frequencies $v_{1}$ and $v_{2}$. The MAS speed was $10 \mathrm{kHz}$ in (a) and $23 \mathrm{kHz}$ in (b). For each set of $v_{1}$ and $v_{2}$, rf modulation was implemented by updating the amplitude and phase every $1 \mu$ s according to Eqs. (9) and (12). $v_{1}$ and $v_{2}$ were independently incremented with a step of $1 \mathrm{kHz}$ with a range of $v_{1}=20-100 \mathrm{kHz}$ in (a) and $20-120 \mathrm{kHz}$ in (b), and $v_{2}=0-100 \mathrm{kHz}$. The individual 8181 and $10201{ }^{13} \mathrm{C}$ free induction decays (FIDs) were collected and Fourier-transformed.

We found that the decoupling efficiency was relatively high for the first nutation frequency $v_{1}$ greater than $80 \mathrm{kHz}$, and for the second nutation frequency $v_{2}$ larger than zero but much smaller than $v_{1}$, thus within the reach of TPPM. For $v_{2}$ larger than $\sim 0.3 v_{1}$, the decoupling efficiency was degraded.

In Figs. 4(a) and 4(b), spinning-speed dependent recoupling bands are observed. Under MAS, the heteronuclear 
(a)

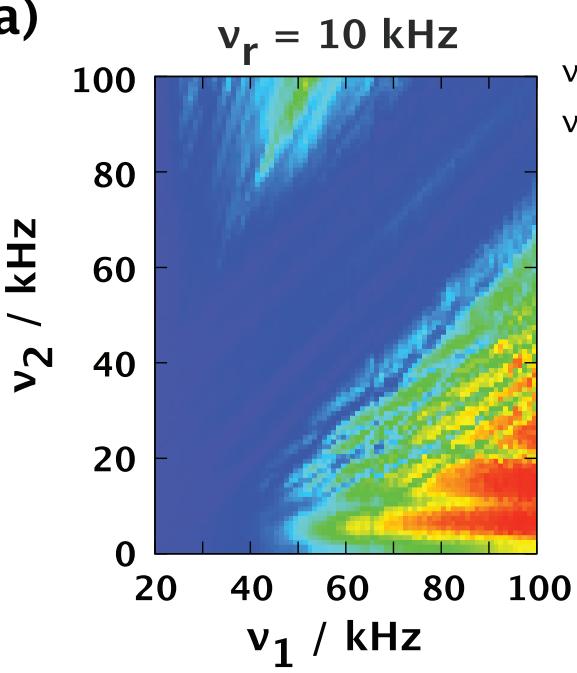

$\mathrm{v}_{1}=100 \mathrm{kHz}$

$v_{2}=14 \mathrm{kHz}$

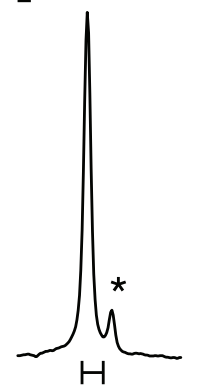

$100 \mathrm{~Hz}$

(b)

$v_{r}=23 \mathrm{kHz}$

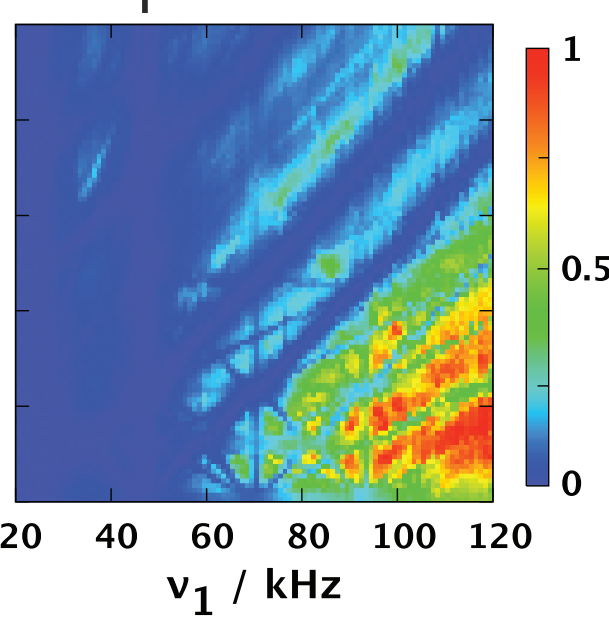

$\mathrm{v}_{1}=100 \mathrm{kHz}$

$v_{2}=19 \mathrm{kHz}$

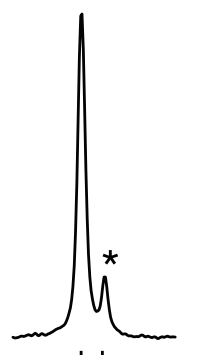

$\mathrm{H}$

$100 \mathrm{~Hz}$
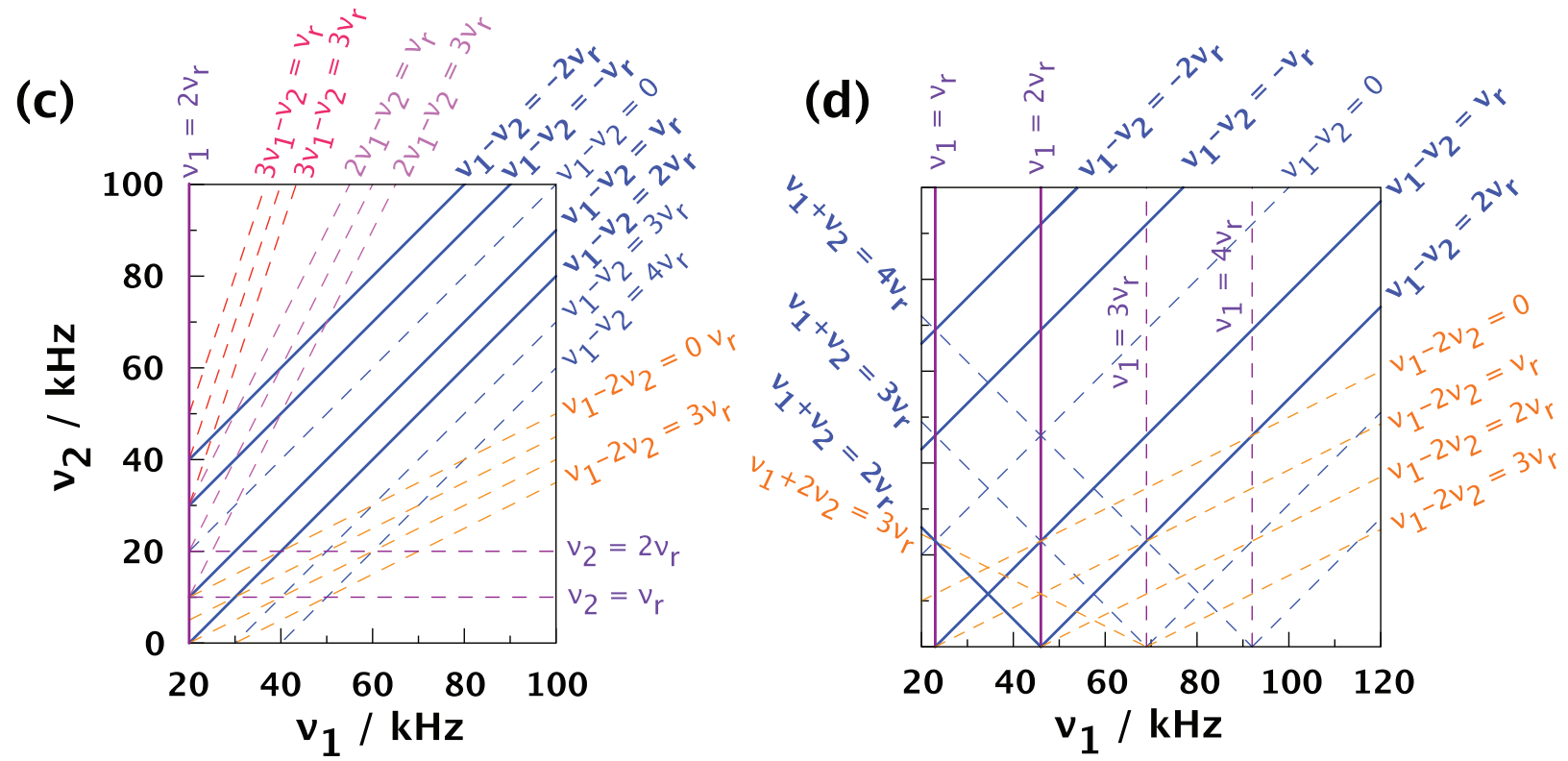

FIG. 4. (a) and (b) Experimental peak heights of the ${ }^{13} \mathrm{C}$ resonance line of the methylene group in polycrystalline $2-{ }^{13} \mathrm{C},{ }^{15} \mathrm{~N}-$ labeled glycine obtained under double-nutation decoupling. The data are plotted as a function of the first and second nutation frequencies $v_{1}$ and $v_{2}$. The spinning speed was $10 \mathrm{kHz}$ in (a), $23 \mathrm{kHz}$ in (b). The nutation frequency was varied with an increment of $1 \mathrm{kHz}$. The peak heights in (a) and (b) are separately normalized by the highest one. ${ }^{13} \mathrm{C}$ resonance lines obtained under the optimal conditions are also shown in (a) and (b), where the asterisks denote irrelevant signals due to a minor crystal polymorph. (c) and (d) Lines of the recoupling bands satisfying $p v_{1}+q v_{2}=r v_{r}$. Those predicted by the first-order analysis (Eqs. (34)-(36)) are depicted with solid lines, while the higher-order recoupling bands are drawn with broken lines.

dipolar interaction $\mathcal{H}_{d}$ is represented as ${ }^{30}$

$$
\begin{gathered}
\mathcal{H}_{d}(t)=D(t) I_{z} S_{z}, \\
D(t)=d\left[\sqrt{2} \sin 2 \beta \cos \left(\gamma+2 \pi v_{r} t\right)\right. \\
\left.-\sin ^{2} \beta \cos \left(2 \gamma+4 \pi v_{r} t\right)\right],
\end{gathered}
$$

where $d$ is the dipolar coupling constant, and $(\beta, \gamma)$ are the Euler angles representing the orientation of the inter-nuclear vector between the ${ }^{1} \mathrm{H}(I)$ and the ${ }^{13} \mathrm{C}(S)$ spins. Under doublenutation irradiation of the $I$ spins, the $I_{z}$ term in the dipolar Hamiltonian is modulated as

$$
\begin{aligned}
=\frac{1}{2}\left\{\sin \left[2 \pi\left(v_{1}-v_{2}\right) t\right]-\sin \left[2 \pi\left(v_{1}+v_{2}\right) t\right]\right\} I_{x}+\sin \left(2 \pi v_{1} t\right) I_{y} \\
+\frac{1}{2}\left\{\cos \left[2 \pi\left(v_{1}-v_{2}\right) t\right]+\cos \left[2 \pi\left(v_{1}+v_{2}\right) t\right]\right\} I_{z} .
\end{aligned}
$$

To the lowest order, interference between the spatial part $D(t)$ and the spin part of the dipolar Hamiltonian is expected for

$$
\begin{gathered}
v_{1}=n v_{r}, \\
v_{1}-v_{2}=n v_{r},
\end{gathered}
$$

and

$$
v_{1}+v_{2}=n v_{r}
$$


with $n= \pm 1, \pm 2$. These conditions exhibit straight lines as plotted in Figs. 4(c) and 4(d) with solid lines. In the experimental results, the clear recoupling bands appear along these lines.

In addition, we found in Figs. 4(a) and 4(b) a number of recoupling bands. They appear on the lines satisfying

$$
k v_{1}+l v_{2}=n v_{\mathrm{r}}
$$

as indicated with broken lines in Figs. 4(c) and 4(d). Such higher-order effects would arise from the cross terms between the ${ }^{1} \mathrm{H}-{ }^{13} \mathrm{C}$ heteronuclear dipolar interaction and the ${ }^{1} \mathrm{H}-{ }^{1} \mathrm{H}$ homonuclear dipolar interactions as well as the anisotropic ${ }^{1} \mathrm{H}$ chemical shielding. In order to confirm this, we carried out numerical simulations in a ${ }^{13} \mathrm{C}-{ }^{1} \mathrm{H}_{2}$ three spin system under double-nutation ${ }^{1} \mathrm{H}$ irradiation, taking account of the ${ }^{1} \mathrm{H}-{ }^{13} \mathrm{C}$ dipolar interactions, ${ }^{1} \mathrm{H}-{ }^{1} \mathrm{H}$ homonuclear dipolar interaction, and anisotropic ${ }^{1} \mathrm{H}$ chemical shifts.

In our simulation, the ${ }^{13} \mathrm{C}-{ }^{1} \mathrm{H}$ distance and the $\mathrm{HCH}$ bond angle was set to $0.091 \mathrm{~nm}$ and $112^{\circ}$, respectively. ${ }^{31} \mathrm{~A}$ symmetric ${ }^{1} \mathrm{H}$ chemical shift tensor with an anisotropy of $14 \mathrm{ppm}$ in the $\delta$ scale was assumed, ${ }^{32}$ corresponding to $5600 \mathrm{~Hz}$ in the magnetic field of $9.4 \mathrm{~T}$ that we used in our experiments. Using a GAMMA library, ${ }^{33}$ we simulated time evolution of the density matrix to obtain the ${ }^{13} \mathrm{C}$ FIDs, which were then Fourier transformed to evaluate the peak heights. For the spinning speed $v_{r}$ of $10 \mathrm{kHz}$, separate simulations were carried out with frequencies $\left(v_{1}, v_{2}\right)$ of double nutation from $v_{1}=20 \mathrm{kHz}$ to $100 \mathrm{kHz}$ and from $v_{2}=0$ to $100 \mathrm{kHz}$ with an increment of $1 \mathrm{kHz} .8181$ peak heights obtained in this way is plotted in Fig. 5(a) as a function of $v_{1}$ and $v_{2}$. For $v_{r}=23 \mathrm{kHz}, v_{1}$ was incremented from 20 to $120 \mathrm{kHz}$ to obtain 10201 FIDs, and the result is plotted in Fig. 5(b).

Even though the model used here was rather simple, the result of the simulation reproduced the features of the recoupling bands observed in the experiments. This indicates that the ${ }^{1} \mathrm{H}$ spins within the methylene group play a major role in the higher-order cross terms to cause the recoupling
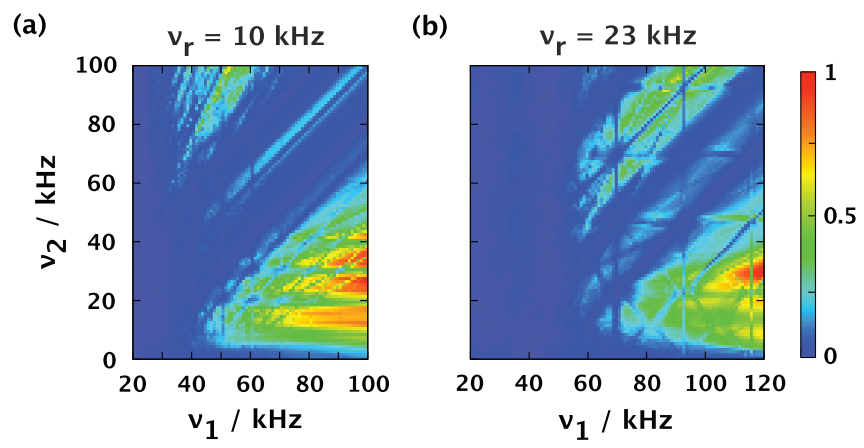

FIG. 5. (a) and (b) Simulated peak heights of the ${ }^{13} \mathrm{C}$ resonance line of the methylene group under double-nutation decoupling and MAS at $10 \mathrm{kHz}$ and $23 \mathrm{kHz}$. A density matrix representing a $\mathrm{CH}_{2}$ three-spin system was employed, and a pair of ${ }^{13} \mathrm{C}-{ }^{1} \mathrm{H}$ dipolar couplings, ${ }^{1} \mathrm{H}-{ }^{1} \mathrm{H}$ dipolar interaction, and ${ }^{1} \mathrm{H}$ chemical shift anisotropies were taken into account. The time dependent Hamiltonian was updated every $2 \mu$ s to simulate time evolution of the density matrix up to $10.24 \mathrm{~ms}$. Powder averaging over 300 Euler angles was performed. bands through the ${ }^{1} \mathrm{H}-{ }^{1} \mathrm{H}$ dipolar interaction as well as the anisotropic ${ }^{1} \mathrm{H}$ chemical shifts. In particular, the latter has a significant effect on the decoupling performance, as pointed out by Scholz et al. in their study on TPPM decoupling. ${ }^{18}$ For comparison, we carried out simulations without taking the ${ }^{1} \mathrm{H}$ chemical shift anisotropies into account, and found that the overall decoupling performance showed unsatisfactory disagreement with the experimental results.

The intervals between the adjacent recoupling bands increases with the spinning speed. Thus, for the slower spinning speed of $10 \mathrm{kHz}$ (Fig. 4(a)), the diagonal region is crowded with the recoupling bands, forming a wide trough. In the case of the moderate but relatively faster spinning speed of $23 \mathrm{kHz}$ (Fig. 4(b)), the recoupling bands spread off, reaching the region of relatively large frequency $v_{1}$ of the first nutation. Care should be taken not to step on one of them in practical applications. Interestingly and importantly, all recoupling bands appear on straight lines in double-nutation decoupling. For a given spinning speed of interest, one may draw straight lines according to Eq. (37), and choose such right-lower region of the $\left(v_{1}, v_{2}\right)$ plane that the recoupling-band lines are well separated.

For comparison, we also carried out TPPM experiments with various rf intensities $v_{\text {rf }}$ and the phase angles $\phi_{0}$, keeping the restriction set by Eq. (1). Results obtained for spinning frequencies of $10 \mathrm{kHz}$ and $23 \mathrm{kHz}$ are shown in Figs. 6(a) and 6(b), where a number of recoupling bands appear as curved lines, in contrast to the case of double-nutation decoupling in which all recoupling bands are on straight lines. Based on the expected equivalence between TPPM and double-nutation, as discussed above at least for small phase angles $\phi_{0}$, we performed the mapping operation by transforming the coordinate lines as described in Fig. 3. Figs. 6(c) and 6(d) show the ${ }^{13} \mathrm{C}$ peak heights plotted using the same data set used in Figs. 6(a) and $6(\mathrm{~b})$, but now as a function of $v_{1}$ and $v_{2}$. Transformation was performed according to Eqs. (29) and (30). For small $\phi_{0}$ and thus small $v_{2}$, the decoupling performance of TPPM and double-nutation is virtually indistinguishable, when we compare the regions of relatively small $v_{2}$ in Figs. 4(a) and 4(b) and Figs. 6(c) and 6(d).

However, for relatively large $\phi_{0}\left(v_{2}\right)$, their equivalence is expected to be broken, so that TPPM irradiation can no longer be described by double nutation. Now, even though the physical meaning of the second nutation frequency $v_{2}$ must be in question, the mapping operation itself is mathematical and can be performed without difficulty. As expected, different profiles of the recoupling bands resulted in the upper regions of Fig. 6. Interestingly, we found that the recoupling bands remain as straight lines which get thinner with increasing $v_{2}$. Avoiding the interference with sample spinning is reminiscent of the R3-robust decoupling techniques such as high-phase TPPM and PISSARRO. Understanding of the spin decoupling dynamics behind this interesting behavior needs further studies.

To summarize, the effect of ${ }^{1} \mathrm{H}$ decoupling under doublenutation irradiation has been studied with arbitrary combinations of the first and second nutation frequencies $v_{1}$ and $v_{2}$. The similarity and difference between double nutation and TPPM under the restriction set by the condition given 

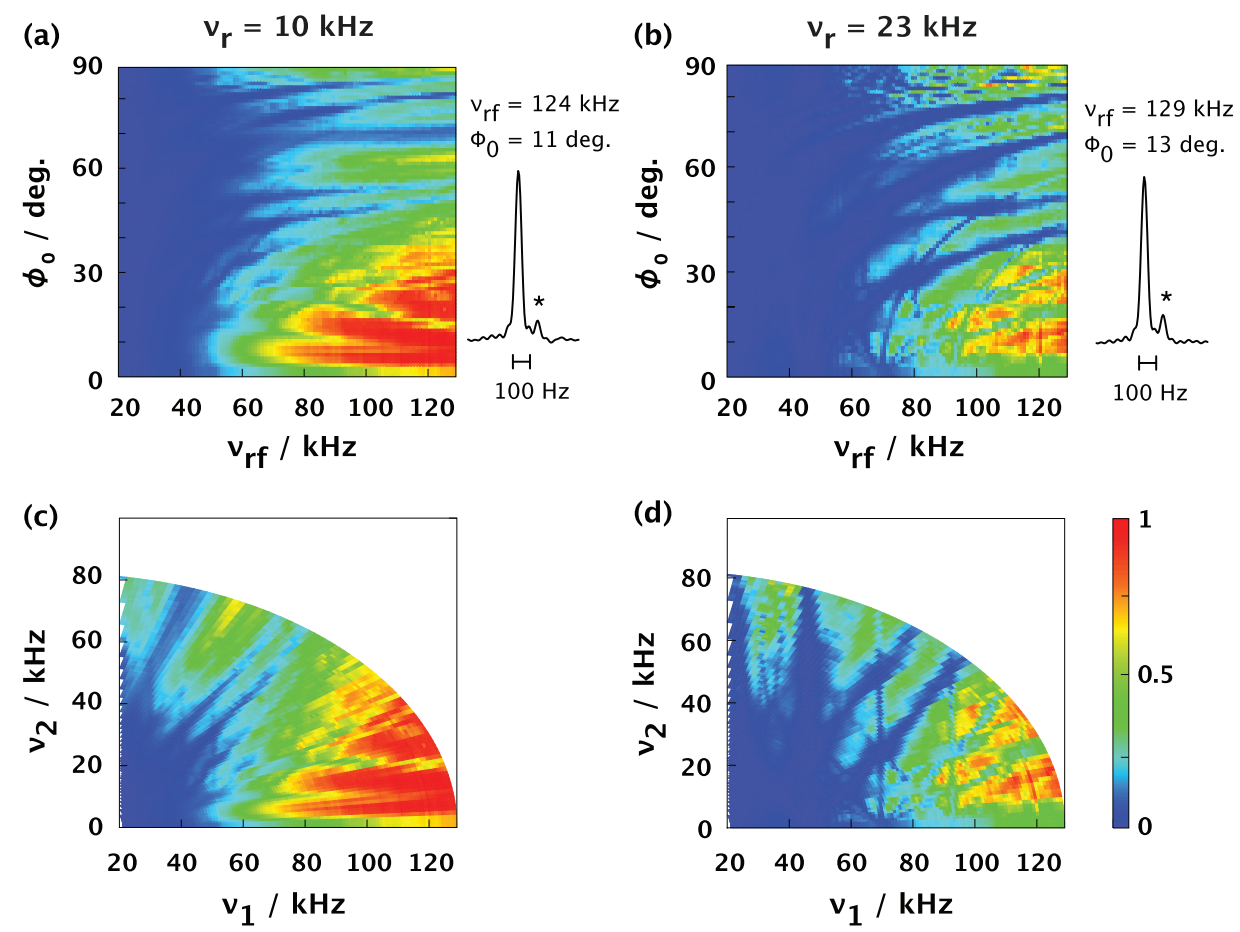

FIG. 6. (a) and (b) Experimental peak height of the ${ }^{13} \mathrm{C}$ resonance line of the methylene group in polycrystalline $2-{ }^{13} \mathrm{C}$, ${ }^{15} \mathrm{~N}$-labeled glycine obtained under TPPM decoupling as a function of the rf amplitude $v_{\text {rf }}$ and the phase angle $\phi_{0}$. The spinning speed was $10 \mathrm{kHz}$ in (a) and $23 \mathrm{kHz}$ in (b). The rf amplitude was varied from $20 \mathrm{kHz}$ to $129 \mathrm{kHz}$ with a step of $1 \mathrm{kHz}$. The phase angle was varied from $0^{\circ}$ to $89^{\circ}$ with an increment of $1^{\circ}$. The pulse width $\tau_{\mathrm{p}}$ was set so that the second resonance condition (Eq. (1)) was satisfied. 9900 peak heights in (a) and (b) are separately normalized by the highest one obtained with ( $v_{\mathrm{rf}}$, $\phi_{0}$ ) $=\left(124 \mathrm{kHz}, 11^{\circ}\right)$ and $\left(129 \mathrm{kHz}, 13^{\circ}\right)$ for (a) and (b). The corresponding ${ }^{13} \mathrm{C}$ spectra are also shown in (a) and (b), where the asterisks denote irrelevant signals due to a minor crystal polymorph. In (c) and (d), the same data as those in (a) and (b) are plotted as a function of the first and the second nutation frequencies $v_{1}$ and $v_{2}$ of double nutation according to the transformation rules given by Eqs. (25) and (26).

in Eq. (1) have been discussed. Double nutation with small $v_{2}$ and TPPM with small $\phi_{0}$ showed equivalent decoupling performance, whereas discrepancy became pronounced as increasing $v_{2}$ and $\phi_{0}$. Even though double nutation did not outperform TPPM, we found its interesting feature that the recoupling bands draw straight lines. This was also found to be the case for TPPM, when the $\left(v_{\mathrm{rf}}, \phi_{0}\right)$-dependence is mapped into the double-nutation plane spanned by $\left(v_{1}, v_{2}\right)$. The results shown in this work suggest that, although implementation of double-nutation is somewhat complicated compared to TPPM, $\left(v_{1}, v_{2}\right)$ are natural parameters from the viewpoint of spin decoupling dynamics.

\section{ACKNOWLEDGMENTS}

K.T. thanks Professor Matthias Ernst for critical comments and fruitful discussions on the subject matter of this paper.

${ }^{1}$ M. Ernst, J. Magn. Reson. 162, 1-34 (2003).

${ }^{2}$ P. Hodgkinson, Prog. Nucl. Magn. Reson. Spectrosc. 46, 197-222 (2005).

${ }^{3}$ L. R. Sarles and R. M. Cotts, Phys. Rev. 111, 853-859 (1958).

${ }^{4}$ J. Tegenfeldt and U. Haeberlen, J. Magn. Reson. 36, 453-457 (1979).

${ }^{5}$ K. Saito, C. Martineau, G. Fink, and F. Taulelle, Solid State Nucl. Magn. Reson. 40, 66-71 (2011).

${ }^{6}$ P. Tekely, P. Palmas, and D. Canet, J. Magn. Reson., Ser. A 107, 129-133 (1994).

${ }^{7}$ A. Detken, E. H. Hardy, M. Ernst, and B. H. Meier, Chem. Phys. Lett. 356, 298-304 (2002).
${ }^{8}$ M. Ernst, A. Samoson, and B. H. Meier, J. Magn. Reson. 163, 332-339 (2003).

${ }^{9}$ A. E. Bennett, C. M. Rienstra, M. Auger, K. V. Lakshmi, and R. G. Griffin, J. Chem. Phys. 103, 6951-6958 (1995).

${ }^{10}$ J. M. Vinther, A. B. Nielsen, M. Bjerring, E. R. H. van Eck, A. P. M. Kentgens, N. Khaneja, and N. C. Nielsen, J. Chem. Phys. 137, 214202 (2012).

${ }^{11}$ J. M. Vinther, N. Khaneja, and N. C. Nielsen, J. Magn. Reson. 226, 88-92 (2013).

${ }^{12}$ R. S. Thakur, N. D. Kurur, and P. K. Madhu, Chem. Phys. Lett. 426, 459463 (2006).

${ }^{13}$ Y. Yu and B. M. Fung, J. Magn. Reson. 130, 317-320 (1998).

${ }^{14}$ B. M. Fung, A. K. Khitrin, and K. Ermolaev, J. Magn. Reson. 142, 97-101 (2000).

${ }^{15}$ A. Khitrin and B. M. Fung, J. Chem. Phys. 112, 2392-2398 (2000).

${ }^{16}$ S. Paul, V. S. Mithu, N. D. Kurur, and P. K. Madhu, J. Magn. Reson. 203, 199-202 (2010).

${ }^{17}$ S. Paul, N. D. Kurur, and P. K. Madhu, J. Magn. Reson. 207, 140-148 (2010).

${ }^{18}$ I. Scholz, P. Hodgkinson, B. H. Meier, and M. Ernst, J. Chem. Phys. 130, 114510 (2009).

${ }^{19}$ Z. Gan and R. R. Ernst, Solid State Nucl. Magn. Reson. 8, 153-159 (1997).

${ }^{20}$ K. Takegoshi, J. Mizokami, and T. Terao, Chem. Phys. Lett. 341, 540-544 (2001).

${ }^{21}$ N. Khaneja and N. C. Nielsen, J. Chem. Phys. 128, 015103 (2008).

${ }^{22}$ L. A. Straas $\varnothing$, M. Bjerring, N. Khaneja, and N. C. Nielsen, J. Chem. Phys. 130, 225103 (2009).

${ }^{23}$ M. Weingarth, P. Tekely, and G. Bodenhausen, Chem. Phys. Lett. 466, 247251 (2008).

${ }^{24}$ M. Weingarth, G. Bodenhausen, and P. Tekely, J. Magn. Reson. 199, 23841 (2009).

${ }^{25} \mathrm{M}$. Weingarth, G. Bodenhausen, and P. Tekely, Chem. Phys. Lett. 502, 259265 (2011).

${ }^{26} \mathrm{M}$. Weingarth, J. Trbosc, J.-P. Amoureux, G. Bodenhausen, and P. Tekely, Solid State Nucl. Magn. Reson. 40, 21-26 (2011). 
${ }^{27}$ Y. Ishii and T. Terao, J. Chem. Phys. 109, 1366-1374 (1998).

${ }^{28}$ K. Takeda, Rev. Sci. Instrum. 78, 033103 (2007).

${ }^{29}$ K. Takeda, J. Magn. Reson. 192, 218-229 (2008).

${ }^{30}$ M. M. Maricq and J. S. Waugh, J. Chem. Phys. 70, 3300-3316 (1979).
${ }^{31}$ R. E. Marsh, Acta Crystallogr. 11, 654-663 (1958).

${ }^{32}$ B. Voigtsberger and H. Rosenberger, Phys. Stat. Sol. A 35, K89-K92 (1976).

${ }^{33}$ S. A. Smith, T. O. Levante, B. H. Meier, and R. R. Ernst, J. Magn. Reson., Ser. A 106, 75-105 (1994). 